\title{
Two cases of emotional disorder after middle cerebral artery infarction showing distinct responses to antidepressant treatment
}

This article was published in the following Dove Press journal:

Neuropsychiatric Disease and Treatment

23 May 2014

Number of times this article has been viewed

\section{Kengo Shimoda \\ Mahito Kimura}

Nippon Medical School Department of Psychiatry, Nippon Medical School, Chiba Hokusou Hospital, Chiba, Japan

Correspondence: Kengo Shimoda Nippon Medical School Department of Psychiatry, Chiba Hokuso Hospital, 17I5 Kamagari, Inzai, Chiba 270-1694, Japan

Tel +8I 47699 IIII

Fax +8I 476991926

Email kshimoda@nms.ac.jp
Abstract: Many emotional disturbances such as post-stroke depression (PSD) and emotional incontinence (EI) commonly occur following cerebrovascular events. The efficacy of antidepressants for these conditions has been established but their comorbid treatment has not been well characterized. In the current study, the authors describe two cases of post-stroke emotional dysregulation; one case with EI; and the other with EI complicated by PSD. The authors describe their differential responses to treatment. Case 1 developed EI after an infarct due to occlusion of the penetrating branches of the left middle cerebral artery (MCA). Case 2 developed both PSD and EI after right MCA stem occlusion. Both patients were initially treated with the selective serotonin reuptake inhibitor (SSRI) paroxetine. Case 1 reacted promptly to SSRI treatment. However, Case 2 had only a partial response to paroxetine, even after many months of treatment. Adjunctive therapy with low-dose aripiprazole was eventually added, resulting in complete improvement of both EI and PSD after 2 additional months of treatment. Thus, Case 2 required a different treatment strategy than Case 1. These findings suggest that aripiprazole adjunctive therapy could be effective for some complex post-stroke emotional disorders.

Keywords: post-stroke disorder, post-stroke depression, emotional incontinence, antidepressants, aripiprazole adjunctive therapy

\section{Introduction}

Emotional disorders are common in post-stroke patients. Many studies have examined the effects and treatment of post-stroke depression (PSD). ${ }^{1}$ Patients with left-sided basal ganglia lesions have shown a significantly higher frequency and severity of depression compared with patients with right-sided basal ganglia or thalamic lesions. ${ }^{2}$ Emotional lability - described as emotionalism, pathological laughing, crying and emotional incontinence (EI) - are common complications in stroke patients. The prevalence of EI has been reported to be $15 \%-20 \% .^{3}$ A previous study showed that EI was frequent in patients with small lenticulocapsular strokes, and was often associated with lesions affecting the dorsal rather than ventral part of the globus pallidus. ${ }^{4}$ Infarcts of the corona radiata are common in the regions supplied by the small blood vessels of the middle cerebral artery (MCA). Thus, ischemic injury involving the internal capsule and basal ganglia seems to be associated with emotional disorders as a result of a stroke. The pathogenesis of post-stroke emotional disorders can involve damage to the biogenic amine pathways, such as serotonin projections that play an important role in the modulation of mood. ${ }^{2}$

Controlled studies of cases with post-stroke emotional disorders (EI and PSD) have examined the effectiveness and tolerability of selective serotonin reuptake 
inhibitors (SSRIs) such as citalopram, fluoxetine, and paroxetine..$^{5-7}$ Many patients with EI have shown a rapid response to low-dose SSRI treatment. Based on evidence of intolerance or poor treatment response to SSRIs, it has been reported that a change in lamotrigine dosage has been effective. ${ }^{8}$ A rapid response to mirtazapine was observed in a study where subjects with both EI and PSD failed to respond to SSRIs. ${ }^{9}$ Moreover, in a double-blind study using nortriptyline for EI, the non-PSD group showed greater improvements in EI compared with the PSD group. ${ }^{10}$ As a result, it appears that improvements in PSD are not directly associated with improvements in EI. To the current authors' knowledge, studies on the treatment of complex emotional disorders such as comorbid EI and PSD following stroke did not include the use of adjunctive therapy. The current study reports two cases of emotional disorders following corona radiata infarct that required differential treatment, thereby suggesting that low-dose aripiprazole adjunctive therapy could be effective in some patients with complex emotional disorders.

\section{Case I}

A 60-year-old woman being treated for hypertension developed dysarthria, right hemiplegia, and tingling. As a result, a diagnosis of acute phase ischemic stroke in the perforating arteries of the MCA was made. Evidence of a single infarct of the left corona radiata was observed on magnetic resonance imaging (MRI) taken the next day (Figure 1). The patient had no history of psychiatric illness such as depression on presentation, and there was no history of psychiatric treatment. Six months after her stroke, the patient displayed daily uncontrollable crying. Although this distressed her caregivers, it was not initially recognized as a psychiatric problem. As a result, the patient only received a psychiatric examination 13 months later. She had been crying for no reason more than ten times a day, but it was also observed that occasionally she could not stop laughing. She described the crying spells as lasting several minutes and stated that she could not resist them. A depressed mood and symptoms consistent with a mood disorder were dismissed following medical examination. The patient had a Hamilton Rating Scale for Depression (HRSD17) ${ }^{11}$ score of 10 points out of a possible $50(10 / 50)$.

To measure the severity of the EI, the current study evaluated the patient using the Pathological Laughter and Crying Scale (PLACS). ${ }^{10}$ A pretreatment PLACS score of 21/27 points suggested moderate disability. Paroxetine $10 \mathrm{mg}$ daily, an SSRI whose efficacy has been supported by previous treatment case reports of EI, was administered. At 2 week follow-up, the subject reported that her crying spells had significantly decreased after initiation of paroxetine, and her PLACS score had decreased from 21 points to 10 points. After 4 weeks of treatment, her PLACS score had decreased to $4 / 27$. At 8 weeks follow-up, she described that her crying spells had ceased. The patient then stopped her hospital visits and
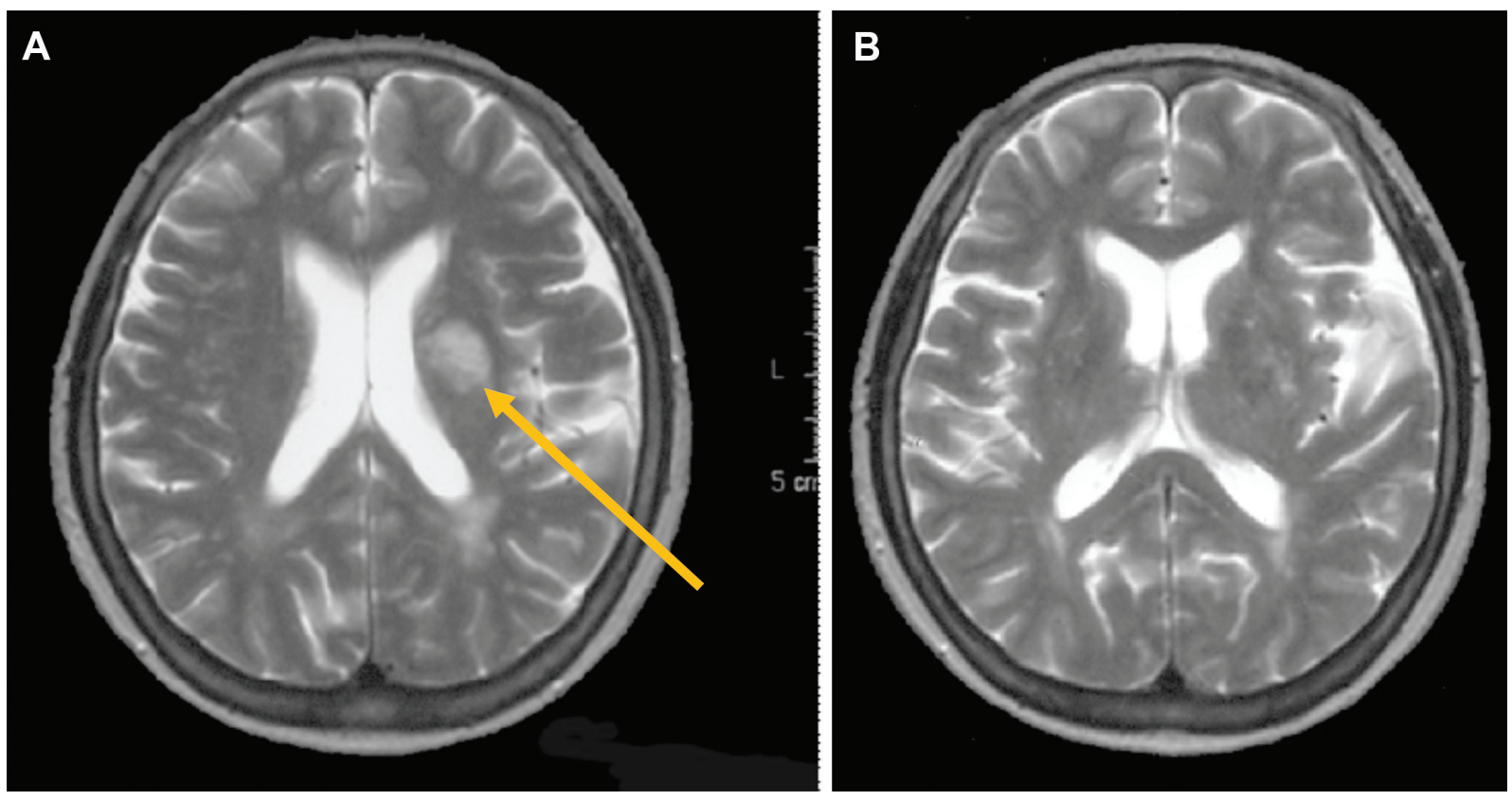

Figure I Axial T2-weighted magnetic resonance imaging (MRI) of the body of the lateral ventricle showing a single infarction in the left corona radiata.

Notes: (A) The arrow points to the major pathological feature. (B) Axial T2-weighted MRI of the basal ganglia showing periventricular hyper-intensity and subcortical white matter hyperintensities in both hemispheres, without major brain infarction. 
discontinued paroxetine. She subsequently had a relapse of crying spells 2 months later accompanied by a PLACS score of $19 / 27$. Treatment with paroxetine $10 \mathrm{mg}$ was restarted and EI was rapidly alleviated. Within 4 weeks, the patient's PLACS score decreased to 2/27. Medication with low-dose paroxetine was continued for 3 months and then treatment was stopped with no signs of recurrence of EI.

\section{Case 2}

A 67-year-old man with a history of depression since age 50 presenting with symptoms of dysarthria and left hemiplegia was admitted to the stroke care unit. An MRI showed an infarct of the right corona radiata as well as infarcts in the caudate nucleus, lentiform nucleus, and right posterior temporal lobe (Figure 2) due to occlusion of the MCA. An MCA stem (M1) occlusion was observed on brain angiography. He showed improvements in blood flow to the MCA after treatment based on acute stroke treatment guidelines; after 30 days, he was transferred to a rehabilitation hospital. Three months after his stroke, he was crying for no reason several times a day and complained of loss of interest and a tendency to be pessimistic. An HRSD17 score of 18/50 suggested that the patient had developed mild to moderate depression and a PLACS score of 20/27 suggested moderate disability.

A score of 28 on the Mini Mental State Examination $(\mathrm{MMSE})^{12}$ did not suggest significant cognitive impairment. As a result, he was diagnosed with comorbid PSD and EI and was started on paroxetine $10 \mathrm{mg}$. Paroxetine was gradually increased to $40 \mathrm{mg}$ over 12 weeks. The PLACS score decreased to $10 / 27$ during the first 4 weeks and HRSD17 decreased to 13 . However, PLACS and HRSD17 scores did not decrease over the next 8 weeks. The left hemiplegia did not improve, even 9 months after his stroke, and he subsequently received superficial temporal artery-to-middle cerebral artery bypass surgery after assessment by cerebral angiography. Administration of paroxetine $40 \mathrm{mg}$ was continued.

He was transferred again to the rehabilitation hospital, where his clinical symptoms improved after surgery. His MMSE score did not decrease but his PLACS and HRSD17 scores did not decrease. In addition, unstable emotional thinking, pessimistic irritability, and aggressive behavior were observed. Therefore, with the informed consent of the patient and family, adjunctive therapy with low-dose aripiprazole $3 \mathrm{mg} /$ day was started. Within 4 weeks, uncontrolled crying spells stopped. Aripiprazole was increased to $6 \mathrm{mg} /$ day over 2 weeks, and after 2 more weeks, his depressive symptoms, irritability, and aggressive behavior were relieved, with a HRDS17 score of 7 and sustained clinical improvement in EI. No adverse effects from the aripiprazole were seen. His lower limb remained hemiplegic but the decreased motivation for rehabilitation was markedly improved. PSD and EI remained in remission (PLACS 0; HRDS17 4) after 2 months of adjunctive therapy with aripiprazole.
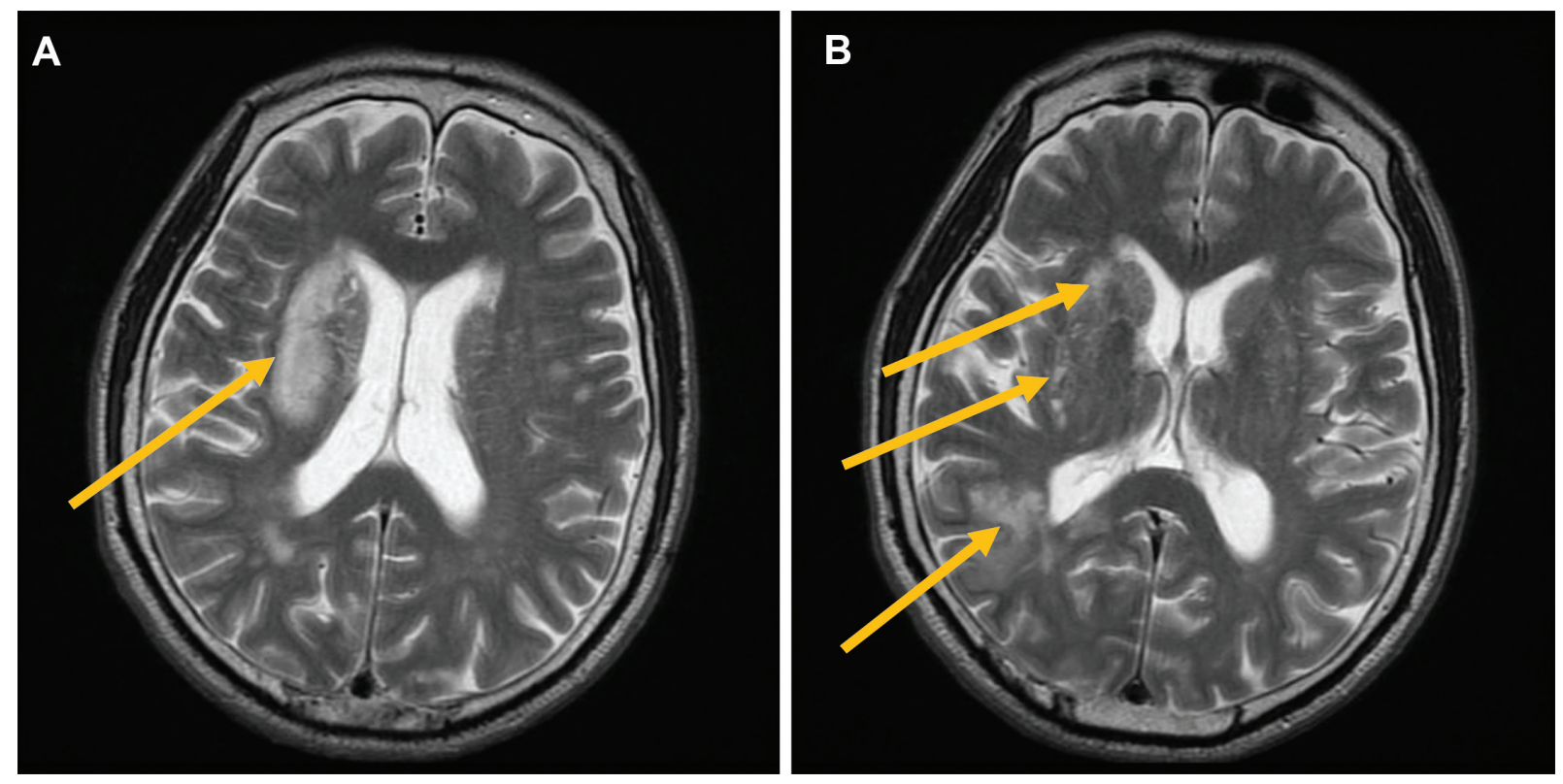

Figure 2 Axial T2-weighted magnetic resonance imaging (MRI) of the body of the lateral ventricle showing infarction of the right corona radiata, without major brain infarction in the left hemisphere.

Notes: (A) Axial T2-weighted MRI of the basal ganglia showing infarction of the caudate nucleus, lentiform nucleus, and right posterior temporal lobe. (B) Subcortical white matter hyperintensities in both hemispheres are present. Arrows point to the major pathological features. 


\section{Discussion}

This study reported two cases of emotional disorder after corona radiata infarct. Case 1 showed post-stroke EI after a single lesion infarct of the left corona radiata who responded rapidly to low-dose SSRI. Case 2 showed both post-stroke PSD and EI after multiple lesions, including a right corona radiata infarct. In Case 2, SSRI treatment showed partial efficacy for the post-stroke EI but was ineffective for the depressive symptoms of PSD. Adjunctive therapy with low-dose aripiprazole was beneficial for the treatment of post-stroke EI and PSD. These results suggest that the course of post-stroke emotional disorders and prognosis for successful treatment after infarction of the MCA can be different in subjects with EI or with a combination of EI and PSD.

In a previous report on the association between PSD and corona radiata infarction, Vataja et $\mathrm{al}^{13}$ reported that in PSD patients, the brain infarct often significantly affected the basal ganglia, mainly the caudate, putamen and pallidum, as well as the posterior corona radiata. They also reported that the frequency of depression was greater in these subjects, with larger infarct size at the sites. However, a clear relationship between specific stroke lesion and development of EI was not established. Previous studies have reported that small lenticulocapsular strokes of the basal ganglia in the right anterior region were closely related to EI. ${ }^{14-16}$ Patients with lenticulocapsular stroke more often develop EI than depression. ${ }^{4}$ The risk factors associated with PSD are: history of depression, increased stroke severity, and post-stroke cognitive or physical impairment. ${ }^{17}$ However, EI does not appear to be related to the presence of motor or general disability. ${ }^{4}$ Most EI patients also have PSD. EI was found to be significantly correlated with mood score and post-stroke depression, as well as with lesion size, activities of daily living (ADL) scores, and intellectual impairment, but not with lesion location, history of stroke, or depression. ${ }^{18}$

Another study found that a past history of depression and cortical lesions were independent predictors of EI. ${ }^{19}$ PSD patients with EI had more severe depressive symptoms compared with patients without comorbid EI. ${ }^{3}$ Therefore, it is thought that the pathologies of post-stroke EI and PSD are partially linked. These previous reports suggest a possible explanation for the two cases in the current study. Case 1 with no history of depression appeared to have pure EI due to a single small corona radiata infarction that was disrupting serotonergic ascending projections. In Case 2, there was a possibility that the activity of other monoamines and serotonin was reduced by multiple infarctions, including the corona radiata, and that history of depression and physical impairment also played an important role in the appearance of both PSD and EI. Starkstein et $\mathrm{al}^{20}$ reported that PSD following MCA lesions was of significantly longer duration than PSD following brainstem and/or cerebellar infarcts. Moreover, ADL were more significantly impaired in patients without PSD than in patients with PSD in the MCA group. ${ }^{20}$ Thus, the delays in recovery from PSD and recovery from physical impairment were interrelated in Case 2.

The most interesting finding in these case reports was that low-dose aripiprazole adjunctive therapy could be effective for post-stroke emotional disorders that were not improving with SSRIs alone. The efficacy of antidepressant treatment for PSD and EI is well-established, and SSRIs are the first-line drugs of choice. Many pure EI cases showed a rapid response to low-dose SSRI treatment as in Case 1. Andersen et $\mathrm{al}^{21}$ investigated the correlation between severity of post-stroke pathological crying with lesion size and location in twelve EI patients. Patients with the most severe EI had relatively large bilateral pontine lesions without lesions in the hemispheres, and clinically least-affected patients had mainly unilateral large subcortical lesions. Therefore, the authors concluded that the destruction of serotonergic raphe nuclei or their ascending serotonergic hemispheric projections could be underlying causes of EI. ${ }^{6}$

This hypothesis is supported by positron emission tomography (PET)-mapping results of high-binding serotonin 5-HT1A receptors in the raphe nuclei of patients with post-stroke EI. ${ }^{22}$ In addition, polymorphisms in the serotonin transporter 5-HTT gene have been shown to be associated with a prevalence of post-stroke EI, with the 5-HTT genelinked promoter region 5-HTTLPR genotype conferring increased susceptibility to EI. ${ }^{23}$ There was rapid improvement in EI symptoms with antidepressant administration but PSD did not improve. The effectiveness of SSRIs for PSD has not been completely established but a more significant effect of nortriptyline compared to fluoxetine has been reported. ${ }^{1}$

A recent study reported on the efficacy and tolerability of adjunctive aripiprazole in patients with treatment-resistant major depression and partial response to antidepressant treatment. ${ }^{24}$ The authors stated that aripiprazole is a second generation antipsychotic with pharmacological properties distinct from other antipsychotics. It acts as a partial agonist at dopamine D2 and D3 and serotonin 5-HT1A receptors, and as an antagonist at 5-HT2A receptors. Activity at these receptors is shared by a number of antidepressant agents, giving aripiprazole a pharmacological rationale for use in depression. ${ }^{24}$

Herrmann et al reported that atypical antipsychotics can be associated with an increased risk of stroke 
in the elderly. ${ }^{25}$ US Food and Drug Administration approved revisions to the safety labeling for aripiprazole warn of the risk of cerebrovascular adverse events, including stroke, associated with its use in elderly patients with dementia-related psychosis. In recognition of these findings, the current study added a low dose of aripiprazole to the case with post-stroke emotional disorder without dementia that had not improved with SSRI treatment, expecting that its dopamine and serotonin agonism would target the symptoms of irritability and aggressive behavior. Severity of depression, other psychopathologies, and neurobiological factors appear to contribute to irritability and aggressive behavior in stroke patients. Moreover, post-stroke irritable and aggressive patients with higher HRSD17 scores responded more favorably to antidepressants.

Although depressed mood was more likely to occur in subjects with emotionalism, most of them were not depressed. ${ }^{26}$ Calvert et $\mathrm{al}^{27}$ reported that post-stroke EI was associated with simple depression, irritability, and ideas of reference among 14 possible psychiatric symptoms derived by aggregating scores from individual items of using a short form of the Present State Examination (PSE) administered during the interview. Post-stroke emotional disorder can have a negative impact on a patient's participation in the rehabilitation process and associated rehabilitation outcomes and be a cause of caregiver stress. ${ }^{2}$ Accordingly, early medical assessment for PSD and appropriate treatment can lead to improved physical and cognitive recovery and decreased mortality.

It is well known that serotonergic and noradrenergic fibers that originate from the brainstem nuclei and innervate the limbic system, prefrontal cortex, and associated structures are involved in the regulation of mood. Furthermore, dopaminergic pathways seem to play a crucial role in mood and depression. The effectiveness of a combination of modafinil, a dopamine agonist, and aripiprazole in a young adult PSD case with aggressive behavior and emotional lability has been reported. ${ }^{28}$ In addition to monoamines, the cholinergic system, through nicotinic acetylcholine receptors, has recently been suggested to be involved in the etiology of major depressive disorders. These pathways can be disrupted by the stroke lesion, which can result in depression and contribute to the complexity of the disorder. ${ }^{29}$

The first-line treatment for PSD are antidepressants but there are exceptions. Dementia was not a factor in the current study, but the risks of using second generation antipsychotics in elderly patients should have been considered. Adjunctive therapy with aripiprazole to minimize sedation can be effective in antidepressant refractory post-stroke emotional disorders, particularly those associated with irritability and aggression. Further studies are required to clarify the efficacy of antipsychotic drugs in complex post-stroke emotional disorders.

\section{Acknowledgment}

We would like to thank Dr Usuda who provided carefully considered feedback and valuable comments.

\section{Disclosure}

The authors report no conflicts of interest in this work.

\section{References}

1. Robinson RG, Schultz SK, Castillo C, et al. Nortriptyline versus fluoxetine in the treatment of depression and in short-term recovery after stroke: a placebo-controlled, double-blind study. Am J Psychiatry. 2000;157(3):351-359.

2. Robinson RG. The Clinical Neuropsychiatry of Stroke: Cognitive, Behavioral and Emotional Disorders following Vascular Brain Injury. 2nd ed. Cambridge, Cambridge University Press; 2006.

3. House A, Dennis M, Molyneux A, Warlow C, Hawton K. Emotionalism after stroke. BMJ. 1989;298(6679):991-994.

4. Kim JS. Post-stroke emotional incontinence after small lenticulocapsular stroke: correlation with lesion location. J Neurol. 2002;249(7):805-810.

5. Müller U, Murai T, Bauer-Wittmund T, von Cramon DY. Paroxetine versus citalopram treatment of pathological crying after brain injury. Brain Inj. 1999;13(10):805-811.

6. Andersen G, Vestergaard K, Lauritzen L. Effective treatment of poststroke depression with the selective serotonin reuptake inhibitor citalopram. Stroke. 1994;25(6):1099-1104.

7. Wiart L, Petit H, Joseph PA, Mazaux JM, Barat M. Fluoxetine in early poststroke depression: a double-blind placebo-controlled study. Stroke. 2000;31(8):1829-1832.

8. Ramasubbu R. Lamotrigine treatment for post-stroke pathological laughing and crying. Clin Neuropharmacol. 2003;26(5):233-235.

9. Kim SW, Shin IS, Kim JM, Lim SY, Yang SJ, Yoon JS. Mirtazapine treatment for pathological laughing and crying after stroke. Clin Neuropharmacol. 2005;28(5):249-251.

10. Robinson RG, Parikh RM, Lipsey JR, Starkstein SE, Price TR. Pathological laughing and crying following stroke: validation of a measurement scale and a double-blind treatment study. Am J Psychiatry. 1993;150(2):286-293.

11. Hamilton M. A rating scale for depression. J Neurol Neurosurg Psychiatry. 1960;23:56-62.

12. Folstein M, Folstein SE, McHugh PR. "Mini-Mental State" a Practical Method for Grading the Cognitive State of Patients for the Clinician. J Psychiatr Res. 1975;12(3):189-198.

13. Vataja R, Pohjasvaara T, Leppävuori A, et al. Magnetic resonance imaging correlates of depression after ischemic stroke. Arch Gen Psychiatry. 2001;58(10):925-931.

14. MacHale SM, O'Rourke SJ, Wardlaw JM, Dennis MS. Depression and its relation to lesion location after stroke. J Neurol Neurosurg Psychiatry. 1998;64(3):371-374.

15. Kim JS, Choi KS. Poststroke depression and emotional incontinence: correlation with lesion location. Neurology. 2000;54(9):1805-1810.

16. Choi KS, Han K, Choi S, et al. Poststroke depression and emotional incontinence: factors related to acute and subacute stages. Neurology. 2012;78(15):1130-1137.

17. Johnson JL, Minarik PA, Nyström KV, Bautista C, Gorman MJ. Poststroke depression incidence and risk factors: an integrative literature review. J Neurosci Nurs. 2006;38(4):316-327. 
18. Andersen G, Vestergaard K, Ingeman-Nielsen M. Post-stroke pathological crying: frequency and correlation to depression. Eur J Neurol. 1995;2(1):45-50.

19. Tang WK, Chan SS, Chiu HF, Ungvari GS, Wong KS, Kwok TC. Emotional incontinence in Chinese stroke patients-diagnosis, frequency, and clinical and radiological correlates. J Neurol. 2004;251(7):865-869.

20. Starkstein SE, Robinson RG, Berthier ML, Price TR. Depressive disorders following posterior circulation as compared with middle cerebral artery infarcts. Brain. 1998;111(Pt 2):375-387.

21. Andersen G, Ingeman-Nielsen M, Vestergaard K, Riis JO. Pathoanatomic correlation between post-stroke pathological crying and damage to brain areas involved in serotonergic neurotransmission. Stroke. 1994;25(5):1050-1052.

22. Møller M, Andersen G, Gjedde A. Serotonin 5HT1A receptor availability and pathological crying after stroke. Acta Neurol Scand. 2007;116(2):83-90.

23. Kim JM, Stewart R, Kang HJ, et al. Associations of serotonergic genes with poststroke emotional incontinence. Int $J$ Geriatr Psychiatry. 2012;27(8):799-806.
24. Marcus RN, McQuade RD, Carson WH, et al. The efficacy and safety of aripiprazole as adjunctive therapy in major depressive disorder: a second multicenter, randomized, double-blind, placebo-controlled study. J Clin Psychopharmacol. 2008;28(2):156-165.

25. Herrmann N, Mamdani M, Lanctôt KL. Atypical antipsychotics and risk of cerebrovascular accidents. Am J Psychiatry. 2004;161(6): 1113-1115.

26. Chan KL, Campayo A, Moser DJ, Arndt S, Robinson RG. Aggressive behavior in patients with stroke: association with psychopathology and results of antidepressant treatment on aggression. Arch Phys Med Rehabil. 2006;87(6):793-798.

27. Calvert T, Knapp P, House A. Psychological associations with emotionalism after stroke. J Neurol Neurosurg Psychiatry. 1998; 65(6):928-929.

28. Capaldi II VF, Wynn GH. Post Stroke Depression: Treatments and Complications in a Young Adult. Psychiatr Q. 2010;81(1):73-79.

29. Loubinoux I, Kronenberg G, Endres M, et al. Post-stroke depression: mechanisms, translation and therapy. J Cell Mol Med. 2012; 16(9):1961-1969.

\section{Dovepress}

\section{Publish your work in this journal}

Neuropsychiatric Disease and Treatment is an international, peerreviewed journal of clinical therapeutics and pharmacology focusing on concise rapid reporting of clinical or pre-clinical studies on a range of neuropsychiatric and neurological disorders. This journal is indexed on PubMed Central, the 'PsycINFO' database and CAS,

and is the official journal of The International Neuropsychiatric Association (INA). The manuscript management system is completely online and includes a very quick and fair peer-review system, which is all easy to use. Visit http://www.dovepress.com/testimonials.php to read real quotes from published authors. 